Casper, Marc; Dilger, Bernadette; Fischer, Frederik; Fütterer, Katharina; Naeve-Stoß, Nicole; Tramm, Tade

\title{
Entwicklung beruflicher Schulen im regionalen Verbund
}

Seifried, Jürgen [Hrsg.]; Seeber, Susan [Hrsg.]; Ziegler, Birgit [Hrsg.]: Jahrbuch der berufs- und wirtschaftspädagogischen Forschung 2017. Opladen ; Berlin ; Toronto : Verlag Barbara Budrich 2017, S. 171-184. (Schriftenreihe der Sektion Berufs- und Wirtschaftspädagogik der Deutschen Gesellschaft für Erziehungswissenschaft (DGfE))

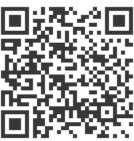

Quellenangabe/ Reference:

Casper, Marc; Dilger, Bernadette; Fischer, Frederik; Fütterer, Katharina; Naeve-Stoß, Nicole; Tramm, Tade: Entwicklung beruflicher Schulen im regionalen Verbund - In: Seifried, Jürgen [Hrsg.]; Seeber, Susan [Hrsg.]; Ziegler, Birgit [Hrsg.]: Jahrbuch der berufs- und wirtschaftspädagogischen Forschung 2017. Opladen ; Berlin ; Toronto : Verlag Barbara Budrich 2017, S. 171-184 - URN:

urn:nbn:de:0111-pedocs-184245 - DOI: 10.25656/01:18424

https://nbn-resolving.org/urn:nbn:de:0111-pedocs-184245

https://doi.org/10.25656/01:18424

in Kooperation mit / in cooperation with:

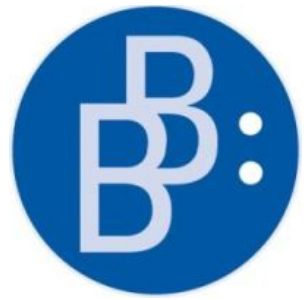

https://www.budrich.de

\section{Nutzungsbedingungen}

Gewährt wird ein nicht exklusives, nicht übertragbares, persönliches und beschränktes Recht auf Nutzung dieses Dokuments. Dieses Dokument ist ausschließlich für den persönlichen, nicht-kommerziellen Gebrauch bestimmt. Die Nutzung stellt keine Übertragung des Eigentumsrechts an diesem Dokument dar und gilt vorbehaltlich der folgenden Einschränkungen Auf sämtlichen Kopien dieses Dokuments müssen alle Urheberrechtshinweise und sonstigen Hinweise auf gesetzlichen Schutz beibehalten werden. Sie dürfen dieses Dokument nicht in irgendeiner Weise abändern, noch dürfen Sie dieses Dokument für öffentliche oder kommerzielle Zwecke vervielfältigen, öffentlich ausstellen, aufführen, vertreiben oder anderweitig nutzen

Mit der Verwendung dieses Dokuments erkennen Sie die Nutzungsbedingungen an.

\section{Terms of use}

We grant a non-exclusive, non-transferable, individual and limited right to using this document.

This document is solely intended for your personal, non-commercial use. Use of this document does not include any transfer of property rights and it is conditional to the following limitations: All of the copies of this documents must retain all copyright information and other information regarding legal protection. You are not allowed to alter this document in any way, to copy it for public or commercial purposes, to exhibit the document in public, to perform, distribute or otherwise use the document in public.

By using this particular document, you accept the above-stated conditions of use.

\section{Kontakt / Contact:}

\section{peDOCS}

DIPF | Leibniz-Institut für Bildungsforschung und Bildungsinformation Informationszentrum (IZ) Bildung

E-Mail: pedocs@dipf.de

Internet: www.pedocs.de

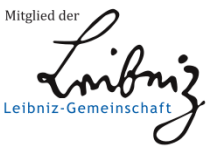


Schriftenreihe der Sektion

Berufs- und Wirtschaftspädagogik

der Deutschen Gesellschaft

für Erziehungswissenschaft (DGfE) 
Jürgen Seifried

Susan Seeber

Birgit Ziegler (Hrsg.)

\section{Jahrbuch der berufs- und} wirtschaftspädagogischen Forschung 2017

Verlag Barbara Budrich

Opladen • Berlin • Toronto 2017 
Bibliografische Information der Deutschen Nationalbibliothek

Die Deutsche Nationalbibliothek verzeichnet diese Publikation in der Deutschen

Nationalbibliografie; detaillierte bibliografische Daten sind im Internet über

http://dnb.d-nb.de abrufbar.

Gedruckt auf säurefreiem und alterungsbeständigem Papier

Alle Rechte vorbehalten

(C2017 Verlag Barbara Budrich, Opladen, Berlin \&Toronto

www.budrich-verlag.de

ISBN 978-3-8474-2141-2 (Paperback)

eISBN 978-3-8474-1131-4 ( eBook)

Das Werk einschließlich aller seiner Teile ist urheberrechtlich geschützt. Jede Verwertung außerhalb der engen Grenzen des Urheberrechtsgesetzes ist ohne Zustimmung des Verlages unzulässig und strafbar. Das gilt insbesondere für Vervielfältigungen, Übersetzungen, Mikroverfilmungen und die Einspeicherung und Verarbeitung in elektronischen Systemen.

Umschlaggestaltung: Bettina Lehfeldt, Kleinmachnow - www.lehfeldtgraphic.de Typographisches Lektorat: Anja Borkam, Jena 


\section{Inhaltsverzeichnis}

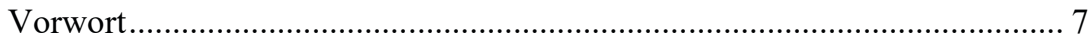

\section{Teil I: Überlegungen zum disziplinären Selbstverständis der Berufs- und Wirtschaftspädagogik}

Karin Büchter

Zum Gehalt berufs- und wirtschaftspädagogischer

Selbstthematisierungen - Rückblick und Ausblick

Matthias Söll

Orientierungspotenziale des Basiscurriculums der Berufs- und

Wirtschaftspädagogik für die disziplinäre Binnen- und Außenlegitimität.... 29

\section{Teil II: Berufliche Lehr-Lern- und Unterrichtsforschung}

Christoph Helm, Jacqueline Netzthaler und Bettina Kreuzer

Kooperatives Lernen im kaufmännischen Unterricht. Eine

Netzwerkanalyse zu sozial-konstruktivistischen Lerntheorien 43

Manuela Niethammer und Anke Langner

Inklusion als fachdidaktischer Anspruch

Svenja Ohlemann und Katja Driesel-Lange

Individuelle Begleitung beruflicher Entwicklung: Kompetenzförderung anhand von Lernstilen.

Mandy Hommel, Bärbel Fürstenau, Claudia Leopold, Héctor Ponce und Mario López

Beitrag von Banken-Webseiten zur Entwicklung der Finanzkompetenz

potentieller Darlehensnehmer/innen über Baufinanzierungen

\section{Teil III: Hochschul- und Lehrerbildungsforschung}

Silke Lange und Dietmar Frommberger

Zur Ausgestaltung schulischer Praxisphasen im beruflichen

Lehramtsstudium - Ergebnisse einer ersten Analyse. 
Heike Jahncke und Karina Kiepe

Handlungsempfehlungen aus dem Einsatz und der Evaluation eines

Tagungsportfolios im Rahmen der Lehrerbildung

Julia Warwas und Andreas Rausch

Unterrichtliche Überzeugungen und Praktiken von Lehrkräften an

Beruflichen Oberschulen - eine fächervergleichende Analyse....

Christian Schmidt

Die Öffnung des Hochschulzugangs für beruflich Gebildete: Förderung studienrelevanter Schlüsselkompetenzen in der Studieneingangsphase am Beispiel der Universität Kassel

\section{Teil IV: Schulentwicklungsforschung}

Marc Casper, Bernadette Dilger, Frederik Fischer, Katharina Fütterer, Nicole Naeve-Stoß und Tade Tramm

Entwicklung beruflicher Schulen im regionalen Verbund.....

Herausgeberschaft.

Autorinnen und Autoren 


\section{Entwicklung beruflicher Schulen im regionalen Verbund}

Marc Casper, Bernadette Dilger, Frederik Fischer, Katharina Fütterer, Nicole Naeve-Stoß und Tade Tramm

\section{Einleitung}

Berufliche Schulen entwickeln sich in Zusammenarbeit in regionalen Bildungsnetzwerken. Das Spektrum reicht von verpflichtender Zusammenarbeit bis $\mathrm{zu}$ projektbasierter, freiwilliger Kooperation. Ziel des Beitrages ist es, Chancen und Herausforderungen von Schulentwicklung (SE) an beruflichen Schulen in Verbünden anhand von zwei Projekten aufzuzeigen. Dazu werden in Anlehnung an das Drei-Wege-Modell der Schulentwicklung nach Rolff (1998) die Ebenen der pädagogisch-didaktischen, der personellen sowie der organisatorisch-institutionellen SE als Aufgabenfeld der Berufs- und Wirtschaftspädagogik thematisiert und die Dimensionen der SE im Verbund im Vergleich zu den SE-Dimensionen der Einzelschule erweitert.

Die kooperativ-schulübergreifende und curricular-didaktische SE wird aus „KaBueNet“, dem Netzwerk der Berliner Oberstufenzentren zur Entwicklung des Curriculums für den/die Kaufmann/-frau für Büromanagement, vorgestellt. Der Zusammenhang von profilierter regionaler Bildungsangebotsgestaltung und institutioneller Entwicklung von Verbünden wird am Beispiel des „Regionalen Berufsbildungszentrums (RBZ) Dortmund“ verdeutlicht. Die Gegenüberstellung und Analyse der beiden Projekte erfolgt u. a. unter Bezugnahme auf Aspekte der Educational Governance mit Blick auf Motive und Impulse für die Projektinitiative, die Konstellationen sowie die Chancen, Herausforderungen und Grenzen der Verbundarbeit.

\section{Das Projekt „KaBueNet“}

\subsection{Motivation und Ausgangslage}

Zum Schuljahr 2014 wurde der neue Ausbildungsberuf ,Kaufmann-/frau für Büromanagement" eingeführt, in dem sich die vorigen bürowirtschaftlichen Berufe vereinen. Mit einem Jahr Vorlauf begannen sieben Berliner Oberstu- 
fenzentren unter wissenschaftlicher Begleitung mit der Entwicklung eines gemeinsamen kompetenzorientierten Lehrplans für den Berufsschulunterricht. Dieser regionale Verbund namens „KaBueNet“ (vgl. www.kabuenet. de) stellt sich dabei mehreren Herausforderungen (vgl. Casper 2016, 3f.):

1. Die Bewältigung der Neuordnung ist das vorrangige Problem aus der Sicht der Lehrenden, die inhaltlich und pragmatisch für den laufenden Unterricht handlungsfähig sein wollen.

2. Die Einführung des Lernfeldkonzepts ist für alle Beteiligten eine zusätzliche Herausforderung, da der neue Beruf erstmals nach Lernfeldern statt nach Fächern gegliedert ist. Die Interpretationsnotwendigkeit des neuen, offeneren Rahmenlehrplans ermöglicht eine eigenständigere curriculare Entwicklung auf Seiten der Schulen, fordert diese aber eben auch ein. Von den Kollegien an den einzelnen Schulen wird eine erhöhte curricular-didaktische Kompetenz gefordert, die eine „Neudefinition didaktischer Arbeit“ (Sloane 2002, 10) darstellt. Diese Kompetenzen können aus dem Kontext traditioneller Lehrarbeit heraus nicht selbstverständlich als vorhanden angenommen werden (vgl. Tramm \& Naeve-Stoß 2016, 50; Straßer 2013, 497).

3. Die Entwicklung einer Kooperationskultur ergibt sich als dritte Problemebene. Schulen lassen sich traditionell eher als „lose gekoppelte Systeme“ (Bormann 2000, 3) kennzeichnen. Im Fachunterricht können Lehrende als Experten eines Fachs relativ autonom unterrichten, wohingegen im fachübergreifenden Lernfeldunterricht eine höhere Abstimmung zwischen den Beteiligten notwendig ist. Das Lehrerteam wird im Lernfeldkonzept zur zentralen Instanz (vgl. Casper 2016, Straßer 2013, Sloane 2003).

In diesem Sinne können die genannten Herausforderungen im Rahmen der Neuordnung auch als (1) inhaltliche, (2) didaktisch/konzeptionelle sowie (3) soziale/organisationale Reformdimensionen interpretiert werden. Um diesen Herausforderungen zu begegnen, bietet sich bei Stadtstaaten wie Berlin eine schulübergreifende Kooperation an, als Ergänzung zur schulinternen Kooperation.

\subsection{Organisation und Arbeitsstränge}

Um als Verbund aufzutreten, sind neben gemeinsamen Zielen auch gemeinsame Beschlüsse und ein gewisses Maß an Verbindlichkeit notwendig. Dennoch hat ein Netzwerk wie KaBueNet größere Freiheitsgrade als eine einzelne, hierarchisch organisierte schulische Institution. Nach den Kategorien der (Educational) Governance-Literatur lässt sich KaBueNet als Netzwerk zwischen den Idealtypen Gemeinschaft und Organisation bezeichnen. Für Ka- 
BueNet ist ,auf der schulübergreifenden Ebene durchaus eine realtypische Gemeinschaft zu erkennen [...], die auf der Reziprozität der aufgeteilten Lernfeldentwicklung basiert (dem Austausch von zeitversetzt und arbeitsteilig erstellten Produkten)“ (Casper 2016, 18). Die Arbeitsteilung erfolgt entlang der in Abbildung 1 dargestellten Arbeitsstränge.

Abb. 1: Aufbauorganisation in KaBueNet

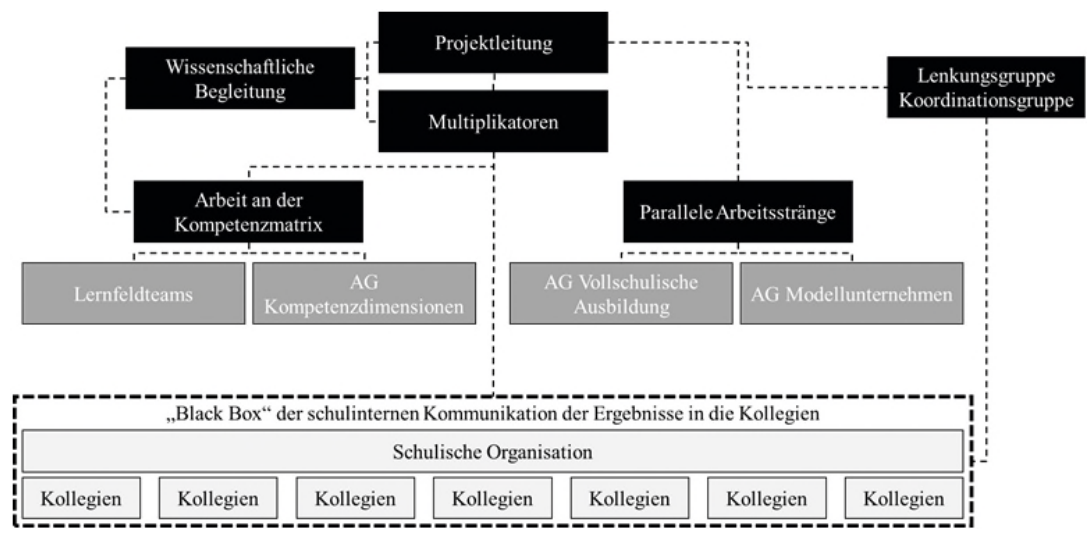

Quelle: Casper $(2016,19)$

Zentraler Bezug der curricularen Entwicklung ist eine Kompetenzmatrix im Sinne des Hamburger Modells. Gemeinsam verabschiedete Gestaltungskriterien sowie Planungsformate bieten somit zwar Orientierung und einen verbindlichen Rahmen, inhaltlich unterliegt die curriculare Arbeit der einzelnen Gruppen jedoch einer projekttypischen Ergebnisoffenheit. Die insgesamt 13 Lernfelder des Bildungsgangs wurden auf die beteiligten Schulen verteilt, sodass im Lernfeld-Arbeitsstrang schulinterne Teams je ein bis drei Lernfelder auf mehreren Planungsebenen ausarbeiten. Eine weitere AG mit jeweils einem Vertreter pro Schule erarbeitet parallel ein System von Kompetenzdimensionen, das als bildungsgangübergreifendes Ordnungsraster zur Entwicklung beruflicher Handlungskompetenz dienen soll. Die beiden Achsen Lernfelder und Kompetenzdimensionen bilden schließlich die Kompetenzmatrix als curriculares Kerninstrument (linker Zweig in Abbildung 1). Parallel hierzu arbeiten schulübergreifende Gruppen an der Ausarbeitung der vollschulischen Variante des Bildungsgangs und an der Entwicklung eines authentischen Modellunternehmens als Lernumwelt (rechter Zweig in Abbildung 1).

Bei der schulübergreifenden Entwicklungsarbeit handelt es sich also um einen Austausch auf Vertrauensbasis, da keine Sanktionen formalisiert wurden und es in KaBueNet keine disziplinierende hierarchische Instanz gibt. Qualitätsambitionen und Erwartungshaltungen können sich allein auf die 
Selbstverpflichtung und das Vertrauen der Beteiligten berufen, was den oben erwähnten Realtypus der Gemeinschaft unterstreicht. Hier zeigt sich ein besonderes Potenzial von Netzwerken für kooperative Bildungsverantwortung, denn ,schwache Verbindungen erhöhen die Wahrscheinlichkeit der Koordination zwischen Akteuren, die sich in Situationen mit einem höheren Anforderungsgehalt nicht aufeinander abgestimmt hätten bzw. gar nicht erst aufeinander getroffen wären" (Kuper 2004, 243-244). Um dieses Aufeinandertreffen zu institutionalisieren und dem Netzwerk eine Projektstruktur zu geben, entwickelte die wissenschaftliche Begleitung ein Workshop-Konzept, in dessen Rahmen bislang 40 schulübergreifende Workshops stattfanden (Stand 31.03.2017). Diese Workshops wurden zu Beginn maßgeblich von der wissenschaftlichen Begleitung gestaltet und sukzessive in die Eigenverantwortung der Projektschulen übertragen, sodass eine langfristige, kontinuierliche Selbststeuerung angestrebt wird.

Auf der Ebene der einzelnen Schulen wiederum entzieht sich das Meiste bislang der Beobachtung der wissenschaftlichen Begleitung (vgl. „Black Box" in der Grafik). Es ist zu erwarten, dass der Erfolg der Implementation an den einzelnen Schulen in besonderem Maße von schulspezifischen organisationalen Faktoren abhängt. Hierauf zielt die Implementationsforschung und -begleitung ab, die aktuell für die kommende Projektphase konzipiert wird.

\subsection{Die Hamburger Kompetenzmatrix als Impulsgeber für Personalentwicklung}

Anhand des Drei-Wege-Modells der Schulentwicklung (vgl. Rolff 1998, 305) wird deutlich, dass Unterrichtsentwicklung (UE), Organisationsentwicklung $(O E)$ und Personalentwicklung (PE) konsequenterweise zusammen zu denken sind. Ausgangspunkt in KaBueNet ist die curriculare Entwicklung von Lernfeldunterricht, das zentrale Anliegen ist also eine Entwicklung des Unterrichts. Hieraus ergeben sich Konsequenzen für die Organisation und das Personal.

Die grundlegende Annahme des Hamburger Modells ist, dass die Ausarbeitung der Lernfelder für ein kompetenzorientiertes Curriculum allein nicht ausreicht. Bei einem rein lernfeldorientierten Lehrplan würden zwar prozessbezogene Kenntnisse, Fertigkeiten und Fähigkeiten ausgebildet. Eine ganzheitliche Entwicklung beruflicher Kompetenzen über die Lernfelder hinweg würde jedoch vernachlässigt, wenn Lernfelder für sich isoliert betrachtet werden. Es bedarf also einer zweiten entwicklungslogischen Achse, die quer $\mathrm{zu}$ allen Lernfeldern mitgedacht werden muss, einer Struktur von Kompetenzdimensionen, die sich aus dem Kompetenzverständnis der Kultusministerkonferenz (KMK-Rahmenlehrpläne) bzw. dem Kompetenzbegriff nach Roth ableitet. Die Dimensionen der „Sach-, Sozial- und Werteinsicht“ (Roth 
1971, 596) bzw. der Fach-, Sozial- und Selbstkompetenz (KMK) müssen für den konkreten Beruf ausdifferenziert und spezifiziert werden. Dies ist eine Reflexion und Zielklärung, die von den Entwicklern und unterrichtenden Kollegien im Rahmen einer schulnahen Curriculumentwicklung geleistet werden muss. Im Fall KaBueNet geschieht dies in der erwähnten schulübergreifenden Arbeitsgruppe „Kompetenzdimensionen“.

\subsection{Zukünftige Projektaufgaben und Herausforderungen}

Zum Sommer 2017 ist der erste Jahrgang beendet, der auf der Basis einer ersten Version der Kompetenzmatrix ausgebildet wurde. Im nächsten Schritt verlagert sich die Netzwerkarbeit somit auf die Evaluation und Überarbeitung auf Basis des bisherigen Unterrichts sowie auf Implementation und Transfer des Curriculums. Aus Sicht der wissenschaftlichen Begleitung ergibt sich der folgende Ausblick für die drei Wege der Schulentwicklung:

- In der Dimension Unterrichtsentwicklung: Iterative Weiterentwicklung und Umsetzung von Lernsituationen im Sinne der Kompetenzmatrix;

- In der Dimension Organisationsentwicklung: Implementierung, Dissemination und Qualitätssicherung in den Einzelschulen;

- In der Dimension Personalentwicklung: Weiterbildung und Coaching der Kollegien an den Einzelschulen durch ausgebildete Multiplikatoren.

\section{Das Projekt „RBZ Dortmund“}

\subsection{Motivation und Ausgangslage}

Das Projekt „Regionales Berufsbildungszentrum (RBZ) Dortmund“ wird in Zusammenarbeit der acht städtischen Berufskollegs, der Stadt Dortmund und des Ministeriums für Schule und Weiterbildung (MSW) des Landes NRW durchgeführt. Im Zeitraum von 2013 bis 2018 wird die Entwicklung und der Aufbau eines Kooperationsverbundes der acht Berufskollegs in verschiedenen Handlungsbereichen der beruflichen Bildung bearbeitet (vgl. Buschfeld, Dilger, Fischer \& Fütterer 2016). Impuls und Auslöser war die Absicht, einen institutionellen Rahmen zu schaffen, um Herausforderungen wie dem grundlegenden Strukturwandel, der demografischen Entwicklung und dem zukünftigen Fachkräftebedarf zu begegnen. Dieser Rahmen sollte dabei der Bedeutung der beruflichen Schule als regionaler Standortfaktor und den besonderen sozioökonomischen Bedingungen einer Großstadt gerecht werden. Intention des Projektvorhabens ist die Entwicklung des strategischen Bildungsange- 
bots, die Entwicklung der institutionellen Rahmenbedingungen sowie einer nachhaltigen Verbund-Arbeit. Die einzelnen Akteure haben unterschiedliche Motivationen, um sich in dem Projekt zu engagieren:

- Durch das Projekt entstehen für das einzelne Berufskolleg Möglichkeiten der Schulentwicklung und der Professionalisierung der eigenen Lehrkräfte.

- Die Gesamtheit der Berufskollegs (als Berufskolleg-Verbund) profitiert durch das Projekt von der Konzentration auf Kooperation (statt auf Konkurrenz), einer effizienten Ressourcennutzung, der Sicherung und Stärkung des Einflusses in der Region (durch das Sprechen mit „einer Stimme") sowie durch die kooperative Lösung dynamischer Anforderungen.

- Die Vorteile für die Stadt Dortmund als Kommune ergeben sich durch eine gemeinsam koordinierte Berufskollegentwicklungsplanung, die Klärung und Zentralisierung von Ansprechpartnern in den Berufskollegs sowie durch ein effizientes Ressourcenmanagement (insb. in Hinblick auf Räumlichkeiten).

- Durch das Projekt werden Alternativen für die Zusammenarbeit beruflicher Schulen entwickelt. Das Projekt stellt somit ein Modellvorhaben für das Land NRW (in Gestalt des Ministeriums für Schule und Weiterbildung) dar.

\subsection{Handlungsfelder des Projekts}

Die Arbeit der einzelnen Projektgruppen ist in Qualitätszirkeln (QZ) organisiert, die sich in vier Handlungsfeldern ordnen. Diese gliedern sich in zwei strategische und zwei operative Handlungsfelder, die sich in ihren Leitideen einerseits mit dem Ziel, Pläne für ein zukünftiges RBZ zu konzipieren, und andererseits bereits jetzt Prozesse unabhängig von der Form des späteren RBZs zu erproben, unterscheiden (siehe Abbildung 2).

Auf der strategischen Ebene werden Konzepte zur Gestaltung, Planung und Steuerung des regionalen Bildungsangebotes entwickelt. Dies führt von der Planung und Umsetzung von Prozessen eines kommunalen Bildungsmonitorings als Datenbasis (QZ Monitoring) zu einer abgestimmten regionalen Bildungsangebotsplanung (QZ regionales Bildungsangebot). Das Handlungsfeld stellt gleichzeitig die Schnittstelle zur Regionalen Berufskollegentwicklungsplanung der Stadt Dortmund (BK-EP) dar. Die BK-EP möchte neben einer Analyse der Ist-Situation und einer Bedarfsabschätzung auf Grundlage der Entwicklung der Schülerzahlen einen Prozess zur Abstimmung und Gestaltung des regionalen Berufsbildungsangebots im Verbund implementieren (vgl. Fischer \& Göckede 2015). In Handlungsfeld II werden Szenarien für eine institutionelle Ausgestaltung der Zusammenarbeit der acht Berufskollegs erarbeitet, mit der Frage, in welcher institutionell-organisato- 
rischen Form das RBZ agieren soll (vgl. Bücking \& Dilger 2015). Es wurden drei Szenarien entwickelt, die sich in ihren jeweiligen Mehrwerten für verschiedene Zielgruppen, ihrem Institutionalisierungsgrad, dem zugrunde liegenden Steuerungsgedanken sowie der Akteurskonstellation unterscheiden: 1) RBZ als Dienstleistungszentrum, 2) RBZ als Modellberufskolleg und 3) RBZ als Dachverband.

Abb. 2: Handlungsfelder des Projekts RBZ Dortmund

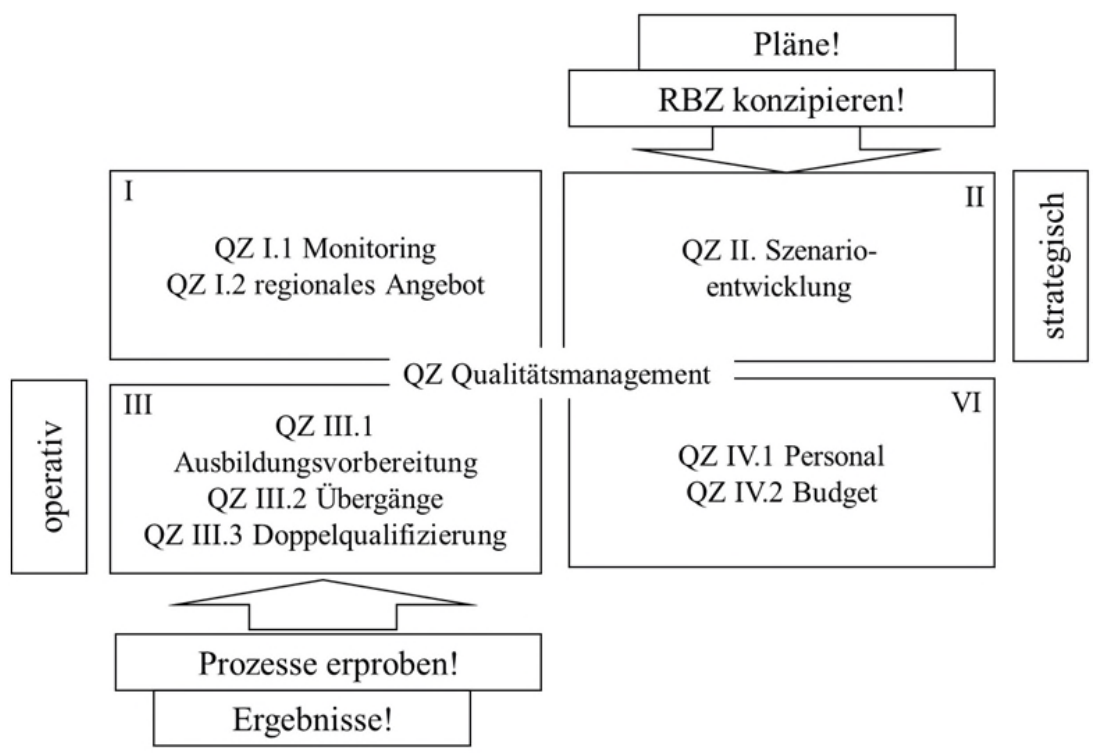

Quelle: Eigene Darstellung nach Buschfeld \& Dilger (2014, 23)

Auf der operativen Ebene werden konkrete schüler- und unterrichtsnahe Prozesse in Zusammenarbeit entwickelt und erprobt (z. B. in der Ausbildungsvorbereitung, der Doppelqualifizierung und der Eingangs- und Übergangsberatung). Es werden dabei Bildungsangebote berücksichtigt, die im Verbund mit Mehrwert entwickelt und umgesetzt werden können. Handlungsfeld IV beschäftigt sich mit der Abstimmung und Erprobung von Personalmanagement- und Beschaffungsprozessen im Verbund. Durch die starke Abhängigkeit der inhaltlichen Ausgestaltung von den institutionellen und rechtlichen Rahmenbedingungen wurde die Arbeit in die Entwicklungsworkshops des Handlungsfeldes II integriert (vgl. Buschfeld \& Dilger 2016, 7). Der QZ Qualitätsmanagement versteht sich als Dienstleister und An- 
sprechpartner für alle Projektteilnehmer und Qualitätszirkel und ist daher keinem Handlungsfeld direkt zugeordnet.

\subsection{Bisherige wissenschaftliche Projektbegleitung und-erfahrung}

Als theoretisch-analytische Referenzkonzepte dienen die Arbeiten zur Schulentwicklung (vgl. Rolff, Buhren, Lindau-Bank \& Müller 2000), zu Qualitätsmanagement beruflicher Schulen (vgl. Dubs 2003) und zu Verbundstrukturen und Educational Governance (vgl. Altrichter, Brüsemeister \& Wissinger 2007; Maag Merki, Langer \& Altrichter 2014). Über die Aktivitäten in den einzelnen Projektjahren wird jährlich ein Bericht der wissenschaftlichen Begleitung veröffentlicht (vgl. Buschfeld \& Dilger 2014; Buschfeld \& Dilger 2015; Buschfeld \& Dilger 2016).

Im Projekt sind verschiedene Spannungsfelder erkennbar geworden. Das Projekt steht auf der strategischen Ebene vor der Herausforderung, dass die Schulen ihre Rolle als alleinstehende Institution verlassen müssen und gefordert sind, eine gemeinsame strategische Zielrichtung zu definieren. Dazu gehört bspw. der Abgleich von Mehrwerten und Aufwänden, die mit dem Verbund für die einzelne Schule verknüpft sind. Auf der operativen Ebene werden die konkreten Herausforderungen der Zusammenarbeit sichtbar, die wiederum im Rahmen der strategischen Ausrichtung zu berücksichtigen sind. Es muss entschieden werden, in welchem Maß Entscheidungsbefugnisse von der Einzelschule an einen Verbund abgegeben werden und wie ein Verbund bei der Steuerung einer Einzelschule mitwirken darf.

\section{Entwicklung beruflicher Schulen im Verbund - eine typisierende Sichtweise}

\subsection{Zur Orientierung}

Beide dargestellten Projekte können in der Tradition einer wirtschaftspädagogischen Gestaltungsforschung gesehen werden, bei der ein Problem der (wirtschaftspädagogischen) Praxis den Ausgangspunkt bildet. Das Erkenntnisinteresse ist dabei von Beginn an auf die systematische Analyse des Problems ausgerichtet, um auf der Grundlage eine theoriegeleitete Weiterentwicklung der Praxis anzuregen (vgl. Sloane 2005; Euler 2011; Dilger 2014). Im Folgenden sollen anhand einer kategorialen Gegenüberstellung der beiden Projekte Gemeinsamkeiten und Unterschiede herausgearbeitet werden, um anschließend auf der Basis der bisherigen Erfahrungen durch die wissen- 
schaftliche Begleitung Herausforderungen, aber auch die Potentiale einer Schulentwicklung im Verbund zu skizzieren.

\subsection{Impulse für die berufliche Schulentwicklung im Verbund}

Herausforderungen für die Entwicklung von beruflichen Schulen im Verbund lassen sich nach exogenen Einflüssen und endogenen Impulsen unterscheiden: Für die beiden skizzierten Projekte können sowohl exogene als auch endogene Entwicklungsimpulse für die berufliche Schulentwicklung im Verbund herausgestellt werden. Während es beim RBZ-Projekt insbesondere um die Institutionalisierung und Professionalisierung der schulischen Organisationsstrukturen und -prozesse geht, wurde das Projekt KaBueNet durch die curriculare Reform in den Büroberufen initiiert. Bei beiden Projekten zeigt sich jedoch das Zusammenspiel der eher pädagogisch-didaktischen mit den organisatorisch-institutionellen Entwicklungsimpulsen. Berufliche Schulentwicklung im Verbund erfolgt demnach in den Bereichen „Entwicklung einer professionellen Bildungsinstitution“ und „Entwicklung profilierter Bildungsangebote".

\subsection{Zielsetzungen der beruflichen Schulentwicklung im Verbund}

Basierend auf den bisherigen Erfahrungen können bei den Zielsetzungen vier unterschiedliche Potentiale und deren Nutzen für die Verbünde identifiziert werden:

- Lern-, Entwicklungs- und Innovationspotentiale können sich daraus ergeben, dass vorhandene Expertisen zusammenkommen, gemeinsam genutzt und weiterentwickelt werden, was sich positiv auf die Qualität von Innovationen auswirken kann - ganz im Sinne: Gemeinsam sind die Schulen entwicklungsfähiger, adaptiver und innovativer als allein.

- Promotorenpotential kann dadurch entstehen, dass Interessen gebündelt und mit Blick auf strategische Ziele zusammen verfolgt werden können.

- Effizienzpotential ist anhand von Synergieeffekten erkennbar, wobei hier vor allem die Optimierung des Ressourceneinsatzes als Mehrwert angesehen werden kann.

- Aus den drei vorgenannten Mehrwerten kann sich im Idealfall ein Autonomiepotential ergeben, denn durch die Arbeit im Verbund können erweiterte Handlungsspielräume des Verbundes entstehen. Neue Innovationen, in Verbindung mit einer Bündelung der Interessen und des Durchsetzungswillens sowie der Optimierungsabsicht bei den eingesetzten Ressourcen, können zu veränderten Rahmenbedingungen 
für die Handlungs- und Gestaltungsspielräume des gesamten Verbunds und/oder auch der Einzelschule führen.

Unserer Erfahrung nach stehen bei den Projekten die Lern-, Entwicklungsund Innovationspotentiale im Vordergrund, gefolgt von den Effizienzpotentialen und den Promotionspotentialen. In der Diskussion im Innenverhältnis der Verbünde hat das Autonomiepotential einen großen Stellenwert. In der Außendarstellung und damit in der Legitimierung der Projekte wird der Mehrwert der gemeinsamen Entwicklung und der damit möglichen Innovationen herausgestellt.

\subsection{Schulentwicklungsdimensionen als Ansatzpunkte für die Klassifikation von Entwicklung beruflicher Schulen im Verbund}

Ausgehend vom Drei-Wege-Modell der Schulentwicklung geht es darum, Unterrichtsentwicklung, Organisationsentwicklung und Personalentwicklung zusammen zu denken (vgl. Rolff 1998, 305). Ein Vergleich der beiden Projekte zeigt, dass im Berliner Projekt die Unterrichtsentwicklung den Ausgangspunkt der Verbundarbeit dargestellt hat und sich daraus Konsequenzen und Entwicklungsherausforderungen für die Dimensionen Personal und Organisation ergeben haben. Im Projekt „RBZ Dortmund“ wurden hingegen im Sinne einer integrierten institutionellen Entwicklung alle drei Entwicklungsdimensionen von Beginn an gleichzeitig bearbeitet. Während sich also beim KaBueNet-Projekt die Frage nach den erforderlichen Entwicklungen auf der Personal- und Organisationsebene zur Sicherstellung der Implementation und Dissemination der curricularen Produkte stellt, entsteht beim RBZ-Projekt die Herausforderung, die unterschiedlichen Entwicklungsaktivitäten auf allen drei Ebenen aufeinander zu beziehen und zusammen zu führen.

\subsection{Merkmale der Educational Governance als Klassifikationspunkte von Entwicklung beruflicher Schulen im Verbund}

Mit der Perspektive der Bildungssteuerung gelangen die Merkmale der Akteure und ihrer Konstellation, die Entwicklung der Mehrebenenlogik zur Steuerung von Bildungsinstitutionen sowie die Formen der kooperativen Arbeitsweise und die Struktur der Arbeitsweise in den Blick (vgl. Kussau \& Brüsemeister 2007). Durch die Form der Zusammenarbeit in beiden Projekten wird eine neue Akteurskonstellation geschaffen, nämlich der Verbund, was $\mathrm{zu}$ einer veränderten Zuweisung von Rechten und Leistungsstrukturen führt. Im RBZ-Projekt stellt sich diesbezüglich als zentrale Frage, wie die Entscheidungsstrukturen im Verbund aufgestellt werden und wie diese mit dem bisher bestehenden Leitungssystem der einzelnen Schule in Abstim- 
mung gebracht werden können. Im Projekt KaBueNet ergeben sich u. a. Fragestellungen hinsichtlich der Verbindlichkeit der entwickelten curricularen Produkte für die Einzelschulen, des Einbezugs und der Beteiligung der Kolleginnen und Kollegen, die nicht am Entwicklungsprozess beteiligt waren und letztlich auch hinsichtlich der Übertragung der Entwicklungsstrategie auf weitere Bildungsgänge.

Mit der Zusammenschließung zu Verbünden bekommt in beiden Projekten der Verbund als Institution eine höhere Bedeutung für die Gestaltung des regionalen Bildungsangebots. Durch die gemeinsame Ausgestaltung und die Etablierung des Verbundes als eigene Akteurkonstellation wird eine weitere Ebene in der Steuerung des regionalen Bildungsangebotes eingeführt. Dies eröffnet einerseits Möglichkeiten für eine spezifischere Profilbildung in der Region und es verbessert die Chancen zur Interessensbündelung und -durchsetzung durch das Wirken des Verbundes als kollektiver Akteur. Andererseits entstehen dadurch Veränderungen in der Abstimmung zwischen den Akteuren in der Berufsbildungsregion. Zudem entsteht als neue Entwicklungsherausforderung die nachhaltige Gestaltung der Beziehungen innerhalb des Verbundes.

\subsection{Zusammenfassende Überlegungen, Potentiale und Herausforderungen der beruflichen Schulentwicklung im Verbund}

In Erweiterung des Drei-Wege-Modells nach Rolff (1998) ergeben sich u.E. angesichts der beruflichen Schulentwicklung in Verbünden folgende drei Dimensionen:

- Entwicklung curricular-didaktischer Konzeptionen,

- Entwicklung eines regional-profilierten Bildungsangebotes,

- Entwicklung von Strukturen und Prozessen mit personellen und institutionellen Regelungen und Kompetenzen im Innen- und Außenverhältnis des Verbundes.

Aus den bisherigen Erfahrungen der wissenschaftlichen Begleitungen in den Projekten können die folgenden Herausforderungen für die Arbeit in regionalen Verbünden festgehalten werden:

- Schaffung einer Balance zwischen gemeinsamen und konkurrierenden Aufgaben sowie Aushandlung von gemeinsamen Interessen und den Interessen der Einzelschulen;

- Sicherstellung der Dissemination und Implementation der im Verbund entwickelten Produkte an den jeweiligen Einzelschulen;

- Motivation der Einzelakteure zur Beteiligung am Verbund, insbesondere dann, wenn der Mehrwert bzw. das Potential der Verbundarbeit nicht 
unmittelbar für den einzelnen Akteur „erfahrbar“ wird, sondern andere Akteure im Verbund entlastet. Und schließlich die

- Entwicklung nachhaltiger Netzwerkstrukturen und -prozesse. In diesem Zusammenhang stellt sich vor allem der hohe Koordinationsaufwand als eine Herausforderung dar, da die Arbeit im Verbund u. a. ein hohes personelles Engagement sowie zeitliche Ressourcen erfordert.

Im Sinne einer ersten Bilanzierung können anhand der bisherigen Erfahrungen folgende Mehrwerte der Arbeit in regionalen Verbünden angenommen werden:

- Größen- und Effizienzeffekte: Durch Entwicklungsarbeiten im berufsschulischen Verbund entsteht die Möglichkeit, Bildungsangebote in der Region zu sichern. Zudem können Effizienzeffekte durch die gemeinsame Entwicklungsarbeit entstehen, insbesondere kann ein Potential bei der didaktischen Konzeptionsentwicklung und in der Etablierung von schülernahen Prozessen festgestellt werden (z. B. bei der Entwicklung doppelqualifizierender Angebote in der dualen Ausbildung).

- Innovationseffekte können durch gemeinsam entwickelte Produkte (z. B. schulische Curricula, Unterrichtsmaterialien) und/oder didaktische Prozessanleitungen (z. B. Handreichungen zur Planung eines lernfeldorientierten Curriculums) entstehen, aber beispielweise auch durch neu konzipierte Bildungsgänge, die an mehreren Einzelschulen angeboten werden.

- Möglichkeiten für Qualitätseffekte ergeben sich durch kollegiale Diskussionen, da ein Austausch z. B. über „gute“ Schule, ,guten“ Unterricht usw. stattfinden und darüber eine Verständigung auf gemeinsame Qualitätskriterien erfolgen kann.

\section{Literatur}

Altrichter, H., Brüsemeister, T. \& Wissinger, J. (2007). Educational Governance: Handlungskoordination und Steuerung im Bildungssystem. Wiesbaden: VS.

Bormann, I. (2000). Schule als lernende Organisation - Kann eine veränderte Lehrerbildung Schule verändern? Sowi-online. Online: http://www.sowi-online.de/sites/ default/files/bormann.pdf (05.01.2017).

Bücking, K. \& Dilger, B. (2015). Entwicklung von regionalen Verbund-Szenarien für berufliche Schulen. Kölner Zeitschrift für Wirtschaft und Pädagogik, 30(59), 2550 .

Buschfeld, D. \& Dilger, B. (2014, 2015, 2016). Weiterentwicklung der acht Berufskollegs in städtischer Trägerschaft zu einem Regionalen Berufsbildungszentrum: Jahresberichte der wissenschaftlichen Begleitung. Online: http://www.rbzdo.de/wissenschaftliche-begleitung (16.12.2016). 
Buschfeld, D., Dilger, B., Fischer, F. \& Fütterer, K. (2016). Weiterentwicklung der Berufskollegs im Verbund - ein Bericht über das Modellprojekt „Regionales Berufsbildungszentrum Dortmund“. bwp@Berufs-und Wirtschaftspädagogik-online, 31, 1-19. Online: http://www.bwpat.de/ausgabe31/buschfeld_etal_bwpat 31.pdf (12.12.2016).

Casper, M. (2016). Schulübergreifende kooperative Curriculumentwicklung aus der Perspektive von organisationalem Lernen, Change Management und Educational Governance am Beispiel des Netzwerks KaBueNet für den Beruf „Kaufmann/frau für Büromanagement".bwp@Berufs- und Wirtschaftspädagogik-online, 31, 1-26. Online: http://www.bwpat.de/ausgabe31/casper_bwpat31.pdf (05.01. 2017).

Dilger, B. (2014). Herausforderungen der Gestaltungsforschung in der Wirtschaftspädagogik. In U. Braukmann, B. Dilger \& H.-H. Kremer (Hrsg.), Wirtschaftspädagogische Handlungsfelder. Festschrift für Peter F. E. Sloane zum 60. Geburtstag, (S. 363-378). Detmold: Eusl.

Dubs, R. (2003). Qualitätsmanagement für Schulen. Studien und Berichte des IWP, 13. St. Gallen: IWP, Institut für Wirtschaftspädagogik.

Euler, D. (2011). Wirkung- vs. Gestaltungsforschung - eine feindliche Koexistenz. Zeitschrift für Berufs- und Wirtschaftspädagogik, 107(4), 520-542.

Fischer, F. \& Göckede, B. (2015). Regionale Berufskollegentwicklungsplanung als schwierig-vereinfachtes Verfahren. Kölner Zeitschrift für Wirtschaft und Pädagogik, 30(59), 3-24.

Kuper, H. (2004). Netzwerke als Form pädagogischer Institutionen. In W. Böttcher \& E. Terhart (Hrsg.), Organisationstheorie in pädagogischen Feldern. Analyse und Gestaltung, (S. 237-252). Wiesbaden: VS Verlag für Sozialwissenschaften.

Kussau, J. \& Brüsemeister, T. (2007). Educational Governance: Zur Analyse der Handlungskoordination im Mehrebenensystem der Schule. In H. Altrichter, T. Brüsemeister \& J. Wissinger (Hrsg.), Educational Governance. Handlungskoordination und Steuerung im Bildungssystem, (S. 14-53). Wiesbaden: Springer VS.

Maag Merki, K., Langer, R. \& Altrichter H. (2014). Educational Governance als Forschungsperspektive: Strategien, Methoden, Ansätze. Wiesbaden: Springer VS.

Rolff, H.-G. (1998). Entwicklung von Einzelschulen: Viel Praxis, wenig Theorie und kaum Forschung - Ein Versuch, Schulentwicklung zu systematisieren. In H.-G. Rolff, K. O. Bauer, K. Klemm \& H. Pfeiffer (Hrsg.), Jahrbuch der Schulentwicklung. Daten, Beispiele und Perspektiven, (S. 295-326). Weinheim: Beltz.

Rolff, H.-G., Buhren, C. G., Lindau-Bank, D. \& Müller, S. (2000). Manual Schulentwicklung: Handlungskonzept zur pädagogischen Schulentwicklungsberatung (SchuB). Weinheim, Basel: Beltz.

Roth, H. (1971). Pädagogische Anthropologie. Hannover: Schroedel.

Sloane, P. F. E. (2002). Schulorganisation und schulische Curriculumarbeit. In R. Bader \& P. F. E. Sloane (Hrsg.), Bildungsmanagement im Lernfeldkonzept. Curriculare und organisatorische Entwicklung. Beiträge aus den Modellversuchsverbünden NELE \& SELUBA, (S. 9-29). Paderborn: Eusl-Verlag.

Sloane, P. F. E. (2003). Schulnahe Curriculumentwicklung. bwp@,Berufs- und Wirtschaftspädagogik - online, 4, 1-23. Online: http://www.bwpat.de/ausgabe4/ sloane_bwpat4.pdf, (05.01.2017). 
Sloane, P. F. E. (2006). Berufsbildungsforschung. In R. Arnold und A. Lipsmeier (Hrsg.), Handbuch der Berufsbildung, (S. 610-627). Wiesbaden: VS Verlag für Sozialwissenschaften.

Straßer, P. (2013). Lernfelder - die (ungenutzte) Wiederkehr des Exemplarischen. Zeitschrift für Berufs- und Wirtschaftspädagogik, 109(4), 496-510.

Tramm, T. \& Naeve-Stoß, N. (2016). Lernfeldübergreifende Kompetenzentwicklung als curriculare Planungsperspektive im Kontext einer kooperativen Curriculumentwicklung in der kaufmännischen Berufsbildung. In A. Dietzen, R. Nickolaus, B. Rammstedt \& R. Weiß (Hrsg.), Kompetenzorientierung. Berufliche Kompetenzen entwickeln, messen und anerkennen, (S. 49-70). Bielefeld: W. Bertelsmann Verlag. 